\title{
Synthesis of New BLnZT Nanostructured Ferroelectric Thin Films
}

\author{
Carlos Ostos $^{*-\dagger}$, Maria L. Martínez-Sarrión ${ }^{\dagger}$, Lourdes Mestres ${ }^{\dagger}$, Alexander Cortés ${ }^{\ddagger}$, Eduardo Delgado ${ }^{\ddagger}$, and Pedro Prieto ${ }^{\ddagger}$ \\ * Grupo de Física de Nuevos Materiales, \\ Universidad Nacional, Bogotá, Colombia \\ ${ }^{\dagger}$ Grupo de Química de Estado Sólido, \\ Departamento de Química Inorgánica, \\ Universitat de Barcelona, Martí i Franquès, \\ 1-11, C.P. 08028, Barcelona, España \\ \#xcellence Center for Novel Materials, Colombia
}

Received on 8 December, 2005

\begin{abstract}
A novel synthesis method was employed to obtain a new family of lead-free compounds with the general formula: $\mathrm{Ba}_{1-y} \mathrm{Ln}_{2 y / 3} \mathrm{Ti}_{0.91} \mathrm{Zr}_{0.09} \mathrm{O}_{3}$ ( $\mathrm{Ln}=$ lanthanide element). The thin films were deposited by RF-magnetron sputtering under high-oxygen pressure on different substrates at $873 \mathrm{~K}$. The crystalline phases were studied via $\mathrm{x}$-ray diffraction, showing the 001 epitaxial reflections corresponding to perovskite single-phase compounds. The films revealed high homogeneity and stoichiometries corresponding to BLnZT ( $\mathrm{Ln}=\mathrm{Nd}$, La). Deposited thin films showed a smooth surface. Ferroelectric measurements through hysteresis curves were obtained in $\mathrm{Ba}_{0.90} \mathrm{La}_{0.067} \square_{0.033} \mathrm{Ti}_{0.91} \mathrm{Zr}_{0.09} \mathrm{O}_{3}$, capacitor structures showing clear ferroelectric behavior with $\mathrm{P}_{r}, \mathrm{P}_{s}$ and $\mathrm{E}_{c}$ of $11.9 \mu \mathrm{C} / \mathrm{cm}^{2}, 36.8 \mu \mathrm{C} / \mathrm{cm}^{2}$ and $38.6 \mathrm{kV} / \mathrm{cm}$, respectively.
\end{abstract}

Keywords: Thin films; Perovskites; Ferroelectrics

\section{INTRODUCTION}

Science and technology of thin films have experienced huge development in the last ten years. The non-volatile ferroelectric random-access memory (NVFRAM) was recently incorporated to commercial products like smart cards and mobile phones. This technology is possible given recent advances in synthesis and characterization of ferroelectric thin films. A future step for science and technology is their integration as capacitors with sub-micrometric sizes to acquire high-density NVFRAM to obtain Gbit capacity nano-capacitors [1].

The hysteric behavior of ferroelectrics related to polarization and electric field utilized for non-volatile memory applications is attractive due to its ability to retain information without requiring an external field; i.e., a back-up battery. One of the critical features of non-volatile memory devices is typically destructive reading, requiring the ferroelectric to endure large amounts of electrical cycles during operation. There have been a number of advances to overcome fatigue in ferroelectric films, such as using oxide electrodes or new alternative systems [2].

$\mathrm{Pb}(\mathrm{Ti}, \mathrm{Zr}) \mathrm{O}_{3}$ (PZT) mixed oxides have been studied given their excellent ferroelectric properties. Research has focused interest on the use of lead-free compounds with characteristics to satisfy technological and environmental requirements. Thus, Perovskite-type mixed oxides derived from $\mathrm{BaTiO}_{3}$ have proven to be promising materials for use as ferroelectric capacitor development $[3,4,5]$.

Thin film growth of $\mathrm{BaTi}_{1-x} \mathrm{Zr}_{x} \mathrm{O}_{3}$ (BZT) materials was carried out via RF-magnetron sputtering. The results show improved dielectric properties for $0.07<x<0.20$ values $[6,7,8]$.

Recently, we have studied the stoichiometric incorporation of lanthanide elements into BZT Perovskite structures. The dielectric properties of these doped materials can modify the bulk behavior, changing the Curie-Weiss transition tempera- ture (Tc) and improving the permittivity $\left(\varepsilon^{\prime}\right)$ with a loss-factor $(\tan \delta)$ near zero for compounds obtained through soft chemistry.

The aim of this work was the synthesis and characterization of new $\mathrm{Ba}_{1-y} \mathrm{Ln}_{2 y / 3} \mathrm{Ti}_{0.91} \mathrm{Zr}_{0.09} \mathrm{O}_{3}$ ( $\mathrm{Ln}=\mathrm{La}, \mathrm{Nd}$ ) Perovskitetype compounds through a novel peroxide-oxalate method. These compounds were employed as targets to obtain a new family of nanostructured ferroelectric thin films.

\section{MATERIALS AND METHODS}

BLnZT compounds were prepared by two different methods. The first one was the oxalate-peroxide method (O-P) based on aqueous solutions of nitrate and chloride precursors [9]. The second one was the conventional ceramic method based on oxide precursors. The structure analysis was carried out through x-ray diffraction (XRD) with a Siemens D-550, Bragg-Brentano configuration, slit 0.05 , rate $5 \mathrm{step} / \mathrm{s}$, radiation $\mathrm{Cu}-\mathrm{K} \alpha$ to $40 \mathrm{kV}$ and $30 \mathrm{~mA}$ between 10 to 100 degrees. Morphological studies were carried out by scanning electron microscopy (SEM) using a JEOL JSM-840 with EDX - AN1000 on samples previously recovered with graphite.

Targets used for thin film growth were obtained at $500 \mathrm{MPa}$ and at $1573 \mathrm{~K}(120 \mathrm{~K} / \mathrm{h})$ for two hours with the corresponding stoichiomtries.

Growth of thin films for the BLnZT ( $\mathrm{Ln}=\mathrm{La}, \mathrm{Nd}$ ) system was made by RF-magnetron sputtering under high-oxygen pressure at $873 \mathrm{~K}$ under the conditions showing in table 1 . The experimental conditions were geared to obtain films with thicknesses between 40 and $100 \mathrm{~nm}$.

Thin film surfaces were analyzed via atomic force microscopy (AFM) with an Autoprobe PC - Park Scientific Instruments, using an $\mathrm{Si}_{3} \mathrm{~N}_{4}$ cantilever between $95-100 \mathrm{nN}$ forces. The AFM images were studied through a SPIPTM program [10]. 
TABLE I: Sputtering conditions for thin film growth.

\begin{tabular}{|l|l|}
\hline $\begin{array}{l}\text { Sputtering } \\
\text { Parameters }\end{array}$ & Conditions \\
\hline Target & $\mathrm{Ba}_{0.90} \mathrm{Ln}_{0.067} \mathrm{Ti}_{0.91} \mathrm{Zr}_{0.09} \mathrm{O}_{3}$ \\
\hline Substrate & $\begin{array}{l}\mathrm{Pt} / \mathrm{TiO}_{2} / \mathrm{SiO}_{2} / \mathrm{Si} \quad(\mathrm{Pt}) ; \mathrm{Si} \quad(100) ; \\
\mathrm{SrTiO}_{3} . \mathrm{Nb} 0.1 \%(\mathrm{STO})\end{array}$ \\
\hline Target-substrate distance & $28 \mathrm{~mm}$ \\
\hline Chamber Atmosphere & $\mathrm{Oxygen}$ \\
\hline Base pressure & $3.4 \mathrm{~Pa}$ \\
\hline Work pressure & $15 \mathrm{~Pa}$ \\
\hline Deposition time & $40 \mathrm{~min}$ \\
\hline RF power & $50 \mathrm{~W}$ \\
\hline BIAS & $400-650 \mathrm{~V}$ \\
\hline Substrate temperature & $873 \mathrm{~K}$ \\
\hline
\end{tabular}

Ferroelectric characterization was carried out using a Radiant Technology System (RT66A) by means of polarization (P) Vs. electric field (E) curves at room temperature.

\section{RESULTS AND DISCUSSION}

The $\mathrm{Ba}_{1-y} \mathrm{Ln}_{2 y / 3} \mathrm{Ti}_{1-x} \mathrm{Zr}_{x} \mathrm{O}_{3}(\mathrm{BLnZT})$ compounds showed a Perovskite single phase for both synthesis methods, but by employing the O-P method, the solid solution range was greater than the ceramic method. This range was defined for lanthanum compounds with values of $\mathrm{La}<0.13$ for any $\mathrm{x}$ value, and $\mathrm{La}<0.17$ for $x<0.15$. In this single Perovskite phase zone, the compounds revealed a cubic structure, $P m 3 m$ (221) - JCPDS 36-0019 [11]. The main impurities found for other $x$ values are $\mathrm{Ba}_{2} \mathrm{TiO}_{4}-\mathrm{JCPDS} 75-0677$ and $\mathrm{La}_{2} \mathrm{Zr}_{2} \mathrm{O}_{7}$ - JCPDS 71-2363. The XRD spectrum corresponding to the $\mathrm{Ba}_{0.90} \mathrm{La}_{0.067} \mathrm{Ti}_{0.91} \mathrm{Zr}_{0.09} \mathrm{O}_{3}$ compound obtained by the O-P method is displayed in Fig. 1.

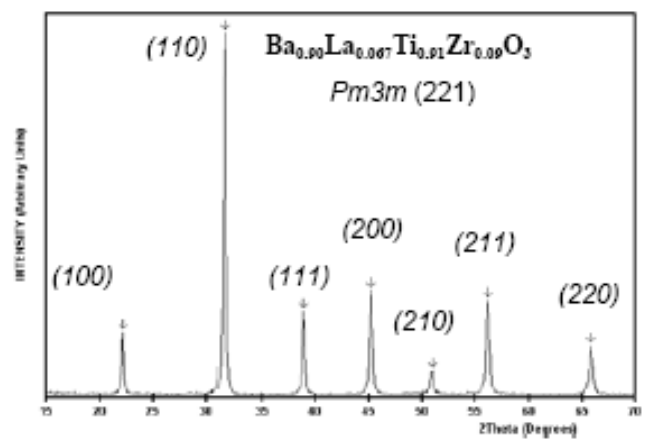

FIG. 1: XRD for a $\mathrm{Ba}_{0.90} \mathrm{La}_{0.067} \mathrm{Ti}_{0.91} \mathrm{Zr}_{0.09} \mathrm{O}_{3}$ Perovskite-type compound obtained by the O-P method.

The BLnZT morphological analysis obtained via SEM indicated important differences for each compound given the presence of the lanthanide element and the synthesis method employed.
Figure 2 displays SEM micrographs of samples obtained by the O-P (left side) and also by the conventional ceramic method (right side) for lanthanide-free BZT compounds and for those containing lanthanide, and neodymium, When this family of compounds was obtained by the ceramic method, grain boundaries were evident, as well as geometrically defined grains. In the same way, lanthanum-doped compounds (BLZT) obtained through both synthesis methods, show surfaces with greater particle size and higher density than BZT compounds. The top-left lanthanum-free sample revealed the highest homogeneity and least surface roughness.

Elemental analysis by EDX on BLnZT compounds shows the qualitative presence of $\mathrm{Ba}, \mathrm{La}, \mathrm{Nd}, \mathrm{Zr}$, and Ti elements on the Perovskite surface. Stoichiometric composition of each compound was corroborated by inductive coupled plasma (AES-ICP).

Furthermore, the results mentioned above confirm the generation of cationic vacancies in the Perovskite structure.open the possibility to improve their dielectric properties, due to the titanium displacement by the distortion of the octahedral sites in the Perovskite structure
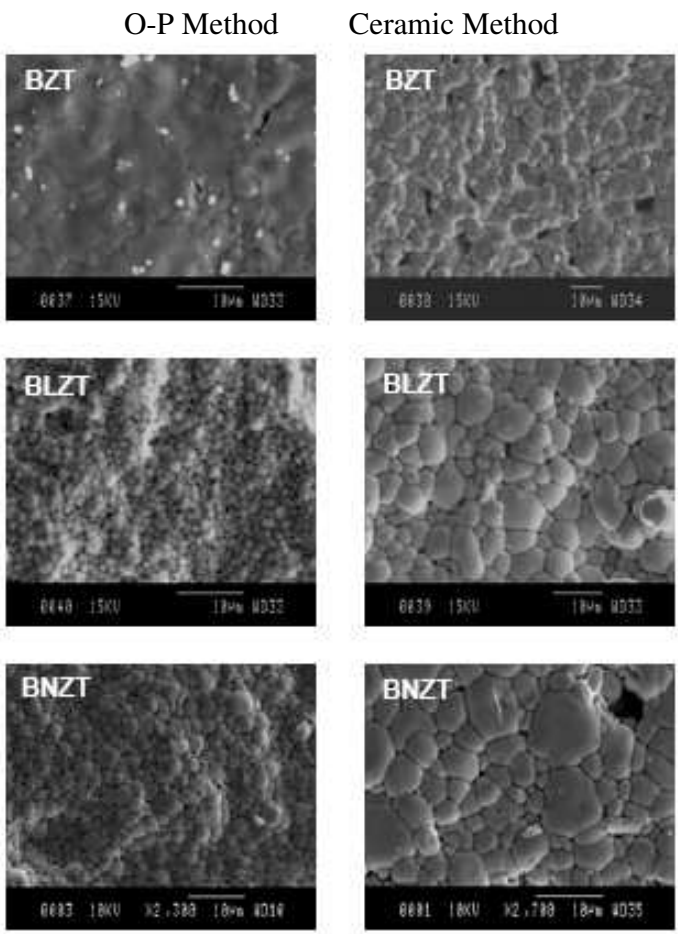

FIG. 2: SEM images for $\mathrm{BaTi}_{0.90} \mathrm{Zr}_{0.10} \mathrm{O}_{3}(\mathrm{BZT})$ and $\mathrm{Ba}_{0.90} \mathrm{Ln}_{0.067} \mathrm{Ti}_{0.91} \mathrm{Zr}_{0.09} \mathrm{O}_{3}(\mathrm{Ln}=\mathrm{La}, \mathrm{Nd})$ compounds obtained by the O-P and conventional ceramic methods.

XRD of a $\mathrm{Ba}_{0.90} \mathrm{Nd}_{0.067} \mathrm{Ti}_{0.91} \mathrm{Zr}_{0.09} \mathrm{O}_{3}(\mathrm{BNZT})$ thin film grown on a $\mathrm{SrTiO}_{3} . \mathrm{Nb}$ (STO) substrate by $\mathrm{RF}$ magnetron sputtering displays the Miller index corresponding to hkl $(00 l)$, showing epitaxial growth with preferential orientation on the $c$ vector (Fig. 3). Best results were obtained on STO substrates, due to cell parameter similarities with BLnZT family compounds; the hkl values correspond to diffraction peaks 
previously reported for BZT thin films [12].

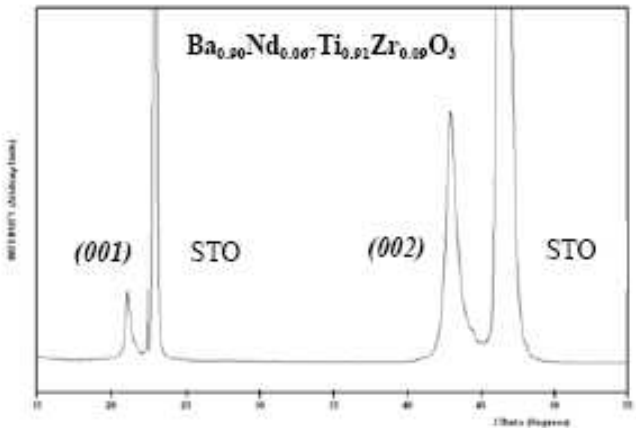

FIG. 3: XRD of BNZT thin film grown on STO substrate.

The uniform growth was confirmed by AFM. For BNZT compounds with four different deposition times (Fig. 4) deposited on Si (100)substrate, under the same experimental conditions previously stated
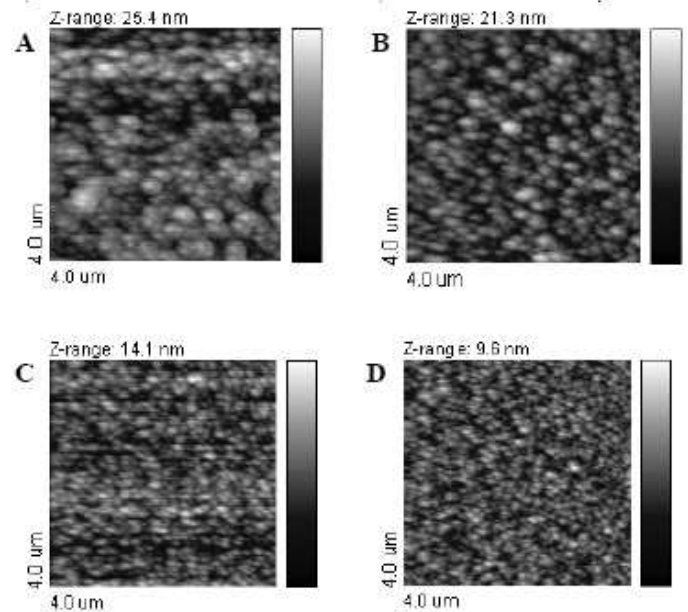

FIG. 4: AFM images of BNZT surfaces of thin films grown on $\mathrm{Si}$ (100)substrates for different deposition times, 15 (A), 30 (B), 45 (C), and 60 (D) minutes.

These images demonstrated continuous growth evolution in BNZT deposition on Si substrates. SPIP ${ }^{\mathrm{TM}}$ software was used to obtain the grain density The results were 10, 13, 20, and 29 grains per $\mu \mathrm{m}^{2}$, corresponding to $15,30,45$, and 60 minutes, respectively.

The tendency mentioned before was directly related to the diminution of the square-average roughness values $\left(\sigma_{R M S}\right)$ with the increase film thickness. The $\sigma_{R M S}$ were $3.42 \mathrm{~nm}$, $2.55 \mathrm{~nm}, 1.81 \mathrm{~nm}$ and $1.41 \mathrm{~nm}$, respectively. The roughness behavior evidenced the reduction of the grain size as follows: $219.1 \mathrm{~nm}, 148.3 \mathrm{~nm}, 141.4 \mathrm{~nm}$, and $114.0 \mathrm{~nm}$ with the increase of the deposition times.

AFM image analysis was useful to deduct differences in the behavior of the thin film grown on each substrate. AFM confirms that the STO material is the best substrate to obtain homogeneous thin films.
The ferroelectric behavior of BLZT ( $\mathrm{L}=\mathrm{La}$ ) thin films was studied by polarization (P)-vs electric field (E) curves (Fig. $5)$. These results were compared to PZT thin film behavior,
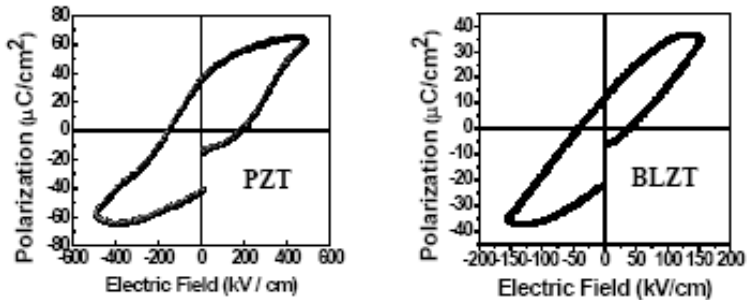

FIG. 5: Hysteresis curves of typical SRO/PZT/Pt (left) and $\mathrm{Ag} / \mathrm{BLZT} / \mathrm{Pt}$ (right) capacitors.

P-E curves show typical SRO/PZT/Pt and Ag/BLZT/Pt capacitors at room temperature. These hysteresis loops displayed asymmetric behaviors due to the asymmetry of electrodes used in both capacitors.

TABLE II: Hysteretic behavior of PZT, BLZT, and BZT thin films

\begin{tabular}{|c|c|c|c|}
\hline & $\mathbf{P}_{r}\left(\mu \mathbf{C} \mathbf{c m}^{-2}\right)$ & $\mathbf{P}_{s}\left(\mu \mathrm{C} \mathbf{c m}^{-2}\right)$ & $\mathbf{E}_{c}\left(\mathbf{k V ~ \mathbf { c m } ^ { - 1 } )}\right.$ \\
\hline PZT & 35.306 & 64.502 & 186.290 \\
\hline BLZT & 11.881 & 36.842 & 38.602 \\
\hline BZT & 5.9 & 13.4 & 45.0 \\
\hline
\end{tabular}

A distinctive feature of the BLZT ferroelectric material is that capacitors with controlled properties, such as those just described for PZT-based capacitors can be fabricated using elemental Ag electrodes. However, the maximum polarization obtained with BLZT-based capacitors is substantially lower than that characteristic of highly oriented PZT-based capacitors, but it is higher than lanthanide-free BZT capacitors reported [13] (Table 2).

\section{CONCLUSIONS}

A new family of Perovskite-type compounds with the general formula $\mathrm{Ba}_{1-y} \mathrm{Ln}_{2 y / 3} \mathrm{Ti}_{0.91} \mathrm{Zr}_{0.09} \mathrm{O}_{3}$ ( $\mathrm{Ln}=\mathrm{La}$, Nd) was synthesized via a novel soft chemistry method and by a conventional ceramic method.

Morphological studies show a direct correlation of insertion of the lanthanide element into the Perovskite structure and the type of synthesis method employed, obtaining surfaces with greater grain size and remarkable grain boundaries for lanthanide-doped compounds obtained by ceramic method.

Moreover, BLnZT thin films were obtained by RFmagnetron sputtering, using the stoichiometric compounds obtained by the O-P method as targets. These thin films were grown on preferential orientation, coinciding with the crystallographic orientation of the STO substrate, which was the best material to achieve smooth and uniform layers with welldefined grains. 
Hysteresis P-E curves displayed clear ferroelectric behavior for Ag/BLZT/Pt capacitors with Ps, Pr values lower than PZT at room temperature, the jump in the hystreresis loop appears due to the different materials used as electrodes. This BLZT environmental-friendly material showed its potential as capacitor material for new electronic and communication technologies

\section{Acknowledgments}

This work was partially supported by Centro de Excelencia en Nuevos Materiales - Colciencias, contract 043-2005; Ministerio de Ciencia y Tecnología, Spain, project BQU 200200619 and Colciencias, contract 1101-0617622.
[1] O. Buffer. Materials with Novel Electronic Properties 6. Academic Press, 2004.

[2] E. Delgado, A. Cortés, W. Lopera, J. Osorio, and P. Prieto. Phys. Stat. Sol. (b), 1-4 (2005)

[3] D. Hennings, R. Waser, U. Weber, G. Greuel, U. Boettger, and S. Weber. J. Amer. Cer. Soc. 84(4), 759 (2001).

[4] Z. Yu, C. Ang, R. Guo, and S. Bhalla. J. App. Phy. 92 (3), 1249 (2002).

[5] D. Wang, R. Yu, S. Fena, W. Zheng, T. Takei, N. Kumuda, and N. Kinomura. Solid State Ionics. 151, 329 (2002).

[6] C-H. Hsi, N-C. Wu, C-H. Chen, and M-C. Wang. J. App. Phy. 94 (1), 598 (2003).

[7] W. S. Choi, J-H. Boo, J. Yi, and B. Hong. Mat. Sci. and Sem.
Proc. 5, 211 (2003).

8] Y. Wang, L. Li, J. Qi, and Z. Gui. Cer. Int. 28, 657 (2002).

[9] S. van der Gijp, L. Winnubst, and H. Verweij. J.Amer. Cer. Soc. 82 (5), 1175 (1999).

[10] SPIPC Image Metrology Lyngby, Denmark, 2002.

[11] N. Wakiya, K. Kuroyanagi, Y. Xuan, K. Shinozaki, and N. Mizutani. Thin Solid Films. 357, 166 (1999).

[12] R. Pantou, C. Dubourdieu, F. Weiss, J. Kreisel, G. Köbernik, and W. Haessler. Mat. Sci. in Sem.Proc. 5, 237 (2003).

[13] S. Halder, S. Bhattacharyya, and S.B. Krupanidhi. Mat. Sci. and Eng. B. 95, 124 (2002). 